\title{
Transition and Transversion on the Common Trinucleotide Circular Code
}

\author{
Emmanuel Benard and Christian J. Michel \\ Equipe de Bioinformatique Théorique, ICube, Université de Strasbourg, CNRS, 300 boulevard Sébastien Brant, 67400 Illkirch, France \\ Correspondence should be addressed to Christian J. Michel; c.michel@unistra.fr
}

Received 15 February 2013; Accepted 22 April 2013

Academic Editor: Alessandra Lumini

Copyright (C) 2013 E. Benard and C. J. Michel. This is an open access article distributed under the Creative Commons Attribution License, which permits unrestricted use, distribution, and reproduction in any medium, provided the original work is properly cited.

\begin{abstract}
In 1996, a trinucleotide circular code which is maximum, self-complementary, and $C^{3}$, called $X_{0}$, was identified statistically on a large gene population of eukaryotes and prokaryotes (Arquès and Michel (1996)). Transition and transversions I and II are classical molecular evolution processes. A comprehensive computer analysis of these three evolution processes in the code $X_{0}$ shows some new results; in particular (i) transversion I on the 2nd position of any subset of trinucleotides of $X_{0}$ generates trinucleotide circular codes which are always $C^{3}$ and (ii) transversion II on the three positions of any subset of trinucleotides of $X_{0}$ yields no trinucleotide circular codes. These new results extend our theory of circular code in genes to its evolution under transition and transversion.
\end{abstract}

\section{Introduction}

We continue our study of properties of trinucleotide circular codes [1-5], trinucleotide comma-free codes $[1,6]$, strong trinucleotide circular codes [7], and the common trinucleotide circular code $X_{0}$ identified in genes [8] (see also the recent statistical analysis by [9]) which could be a translation code [10]. A trinucleotide is a word of three letters (triletter) on the genetic alphabet $\{A, C, G, T\}$. The set of 64 trinucleotides is a code (called genetic code), more precisely a uniform code but not a circular code (see Remark 2). In the past 50 years, codes, comma-free codes, and circular codes have been mathematical objects studied in theoretical biology, mainly to understand the structure and the origin of the genetic code as well as the reading frame (construction) of genes, for example, [11-13]. In order to have an intuitive meaning of these notions, codes are written on a straight line while comma-free codes and circular codes are written on a circle, but in both cases, unique decipherability is required. Circular codes only belong to some subsets of the 64 trinucleotide set while comma-free codes are even more constrained subsets of circular codes [1].

Before the discovery of the genetic code, Crick et al. [11] proposed a maximum comma-free code of 20 trinucleotides for coding the 20 amino acids. This comma-free code turned out to be invalid (see, e.g., [14]). In 1996, a maximum circular code $X_{0}$ of 20 trinucleotides was identified statistically on a large gene population of eukaryotes and also on a large gene population of prokaryotes [8]

$$
\begin{gathered}
X_{0}=\{A A C, A A T, A C C, A T C, A T T, C A G, C T C, \\
C T G, G A A, G A C, G A G, G A T, G C C, G G C, \\
\text { GGT, GTA, GTC, GTT, TAC, TTC }\} .
\end{gathered}
$$

This code $X_{0}$ has remarkable mathematical properties as it is a $C^{3}$ self-complementary maximum circular code (see the following). Since 1996, its properties have been studied in detail by different authors, for example, [9, 15-21]. Transition and transversions I and II are classical molecular evolution processes, for example, [22]. By using an algorithm based on the necklace, we perform here a comprehensive computer analysis of these three evolution processes in the code $X_{0}$. Some new results are identified with the code $X_{0}$ by computer analysis; in particular (i) transversion I on the 2nd position of any subset of trinucleotides of $X_{0}$ generates trinucleotide circular codes which are always $C^{3}$ and (ii) transversion II on the three positions of any subset of trinucleotides of $X_{0}$ yields no trinucleotide circular codes. 


\section{Preliminaries}

The classical notions of language theory and codes can be found in $[23,24]$. Let $\mathscr{A}_{4}=\{A, C, G, T\}$ denote the genetic alphabet, lexicographically ordered by $A<C<G<T$. The set of words (nonempty words, resp.) on $\mathscr{A}_{4}$ is denoted by $\mathscr{A}_{4}^{*}$ $\left(\mathscr{A}_{4}^{+}\right.$, resp.). The set of the 16 words of length 2 (dinucleotides or diletters) on $\mathscr{A}_{4}$ is denoted by $\mathscr{A}_{4}^{2}=\{A A, A C, \ldots, T T\}$. The set of the 64 words of length 3 (trinucleotides or triletters) on $\mathscr{A}_{4}$ is denoted by $\mathscr{A}_{4}^{3}=\{A A A, A A C, \ldots, T T T\}$.

Definition 1. A subset $X \subset \mathscr{A}_{4}^{+}$is a code on $\mathscr{A}_{4}$ if for each $x_{1}, \ldots, x_{n}, x_{1}^{\prime}, \ldots, x_{m}^{\prime} \in X, n, m \geq 1$, the condition $x_{1} \cdots x_{n}=$ $x_{1}^{\prime} \cdots x_{m}^{\prime}$ implies $n=m$ and $x_{i}=x_{i}^{\prime}$ for $i=1, \ldots, n$.

Remark 2. $\mathscr{A}_{4}^{3}$ is a code.

Any nonempty subset of $\mathscr{A}_{4}^{3}$ is a code called here trinucleotide code.

Definition 3. A trinucleotide code $X \subset \mathscr{A}_{4}^{3}$ is circular if, for each $x_{1}, \ldots, x_{n}, x_{1}^{\prime}, \ldots, x_{m}^{\prime} \in X, n, m \geq 1, p \in \mathscr{A}_{4}^{*}, s \in \mathscr{A}_{4}^{+}$, the conditions $s x_{2} \cdots x_{n} p=x_{1}^{\prime} \cdots x_{m}^{\prime}$ and $x_{1}=p s$ imply $n=$ $m, p=\varepsilon$ (empty word) and $x_{i}=x_{i}^{\prime}$ for $i=1, \ldots, n$.

Notation 1. A trinucleotide circular code is noted $C$.

Remark 4. $\mathscr{A}_{4}^{3}$ is not a trinucleotide circular code.

Let $l_{1}, l_{2}, \ldots, l_{n-1}, l_{n}$ be letters in $\mathscr{A}_{4}, d_{1}, d_{2}, \ldots, d_{n-1}, d_{n}$ diletters in $\mathscr{A}_{4}^{2}$, and $n$ an integer satisfying $n \geq 2$.

Definition 5. We say that the ordered sequence $l_{1}, d_{1}, l_{2}$, $d_{2}, \ldots, d_{n-1}, l_{n}, d_{n}, l_{n+1}$ is an $(n+1) \mathrm{LDCN}$ (Letter Diletter Continued Necklace) for a subset $X \subset \mathscr{A}_{4}^{3}$ if

$$
\begin{gathered}
l_{1} d_{1}, l_{2} d_{2}, \ldots, l_{n} d_{n} \in X \\
d_{1} l_{2}, d_{2} l_{3}, \ldots, d_{n-1} l_{n}, d_{n} l_{n+1} \in X .
\end{gathered}
$$

Only a few trinucleotide codes are circular. Two propositions based on the necklace concept allow to determine if a trinucleotide code is circular or not $[2,18]$.

Proposition 6 (see [18]). Let $X$ be a trinucleotide code. The following conditions are equivalent:

(i) $X$ is a trinucleotide circular code;

(ii) X has no $5 \mathrm{LDCN}$.

Definition 7 . We say that the ordered sequence $l_{1}, d_{1}, l_{2}$, $d_{2}, \ldots, d_{n-1}, l_{n}, d_{n}, l_{n+1}$ is an $(n+1)$ LDCCN (Letter Diletter Continued Closed Necklace) for a subset $X \subset \mathscr{A}_{4}^{3}$ if

$$
\begin{gathered}
l_{1} d_{1}, l_{2} d_{2}, \ldots, l_{n} d_{n} \in X, \\
d_{1} l_{2}, d_{2} l_{3}, \ldots, d_{n-1} l_{n}, d_{n} l_{1} \in X .
\end{gathered}
$$

Proposition 8 (see [2]). Let $X$ be a trinucleotide code. The following conditions are equivalent: (i) $X$ is a trinucleotide circular code;

(ii) $X$ has no $n \mathrm{LDCCN}$ for any integer $n \in\{2,3,4,5\}$.

Definition 9. A trinucleotide circular code $X \subset \mathscr{A}_{4}^{3}$ is maximal if, for each $x \in \mathscr{A}_{4}^{3}, x \notin X, X \cup\{x\}$ is not a trinucleotide circular code.

Definition 10. A trinucleotide circular code containing exactly $k$ elements is called a $k$-trinucleotide circular code.

Definition 11. A 20-trinucleotide circular code is maximum as no trinucleotide circular code can contain more than 20 words.

Notation 2. A maximum trinucleotide circular code is noted MC.

Remark 12. A 20-trinucleotide circular code is both maximal and maximum.

We recall two classical genetic maps: complementary and circular permutation.

Definition 13. The complementary genetic map $\mathscr{C}: \mathscr{A}_{4}^{+} \rightarrow$ $\mathscr{A}_{4}^{+}$is defined by

$$
\begin{array}{ll}
\mathscr{C}(A)=T, & \mathscr{C}(C)=G, \\
\mathscr{C}(G)=C, & \mathscr{C}(T)=A
\end{array}
$$

and for all $u, v \in \mathscr{A}_{4}^{+}$by

$$
\mathscr{C}(u v)=\mathscr{C}(v) \mathscr{C}(u)
$$

Example 14. $\mathscr{C}(A C G)=C G T$. This map $\mathscr{C}$ is associated with the property of the complementary and antiparallel double helix (one DNA strand chemically oriented in a $5^{\prime}-3^{\prime}$ direction and the other DNA strand in the opposite $3^{\prime}-5^{\prime}$ direction).

Definition 15. The complementary map $\mathscr{C}$ on a trinucleotide $x$ is naturally extended to a trinucleotide code $X$ as follows:

$$
\mathscr{C}(X)=\left\{y \mid x, y \in \mathscr{A}_{4}^{3}, x \in X, y=\mathscr{C}(x)\right\}
$$

Definition 16. The circular permutation genetic map $\mathscr{P}^{3} \mathscr{A}_{4}^{3}$ $\rightarrow \mathscr{A}_{4}^{3}$ permutes circularly a trinucleotide $l_{1} l_{2} l_{3}, l_{1}, l_{2}, l_{3} \in \mathscr{A}_{4}$, as follows:

$$
\mathscr{P}\left(l_{1} l_{2} l_{3}\right)=l_{2} l_{3} l_{1}
$$

Example 17. $\mathscr{P}(A C G)=C G A$.

Definition 18. The circular permutation map $\mathscr{P}$ on a trinucleotide $x$ is naturally extended to a trinucleotide code $X$ as follows:

$$
\mathscr{P}(X)=\left\{y \mid x, y \in \mathscr{A}_{4}^{3}, x \in X, y=\mathscr{P}(x)\right\} .
$$


Notation 3. The $k$ th iterate of $\mathscr{P}$ is denoted by $\mathscr{P}^{k}$.

Remark 19. The trinucleotide codes $\mathscr{P}(X)$ and $\mathscr{P}^{2}(X)$ are the conjugated classes of the trinucleotide code $X$.

Definition 20. A trinucleotide circular code $X$ is selfcomplementary if, for each $x \in X, \mathscr{C}(x) \in X$.

Notation 4. A self-complementary trinucleotide circular code is noted SC.

Remark 21. A $k$-trinucleotide circular code for $k$ odd cannot be self-complementary.

Definition 22. A trinucleotide circular code $X$ is $C^{3}$ if $X$, $\mathscr{P}(X)$, and $\mathscr{P}^{2}(X)$ are trinucleotide circular codes.

Notation 5. A $C^{3}$ trinucleotide circular code $X$ is noted $C^{3}$.

Definition 23. A trinucleotide circular code $X$ is $C^{3}$ selfcomplementary maximum if $X$ is maximum, $X=\mathscr{C}(X)$ (self-complementary), and $\mathscr{P}(X)$ and $\mathscr{P}^{2}(X)$ are trinucleotide circular codes satisfying $\mathscr{C}(\mathscr{P}(X))=\mathscr{P}^{2}(X)$.

Notation 6. A $\mathrm{C}^{3}$ self-complementary maximum circular code is noted $\mathrm{MSC}^{3}$.

The set $X_{0}$ of 20 trinucleotides identified in the gene populations of both eukaryotes and prokaryotes is a $C^{3}$ selfcomplementary maximum circular code $\mathrm{MSC}^{3}$ [8]; that is, $X_{0}$ is maximum, $X_{0}=\mathscr{C}\left(X_{0}\right), \mathscr{P}\left(X_{0}\right)=X_{1}$, and $\mathscr{P}^{2}\left(X_{0}\right)=X_{2}$ are trinucleotide circular codes, and $\mathscr{C}\left(X_{1}\right)=X_{2}$.

We recall three classical evolution genetic maps: transition and transversions I and II, for example, [22] and extend their definitions to the positions of a trinucleotide.

Definition 24. The transition evolution genetic map $\mathscr{T}$ : $\mathscr{A}_{4}^{+} \rightarrow \mathscr{A}_{4}^{+}$is defined by

$$
\begin{array}{ll}
\mathscr{T}(A)=G, & \mathscr{T}(C)=T, \\
\mathscr{T}(G)=A, & \mathscr{T}(T)=C .
\end{array}
$$

Definition 25. The transition map $\mathscr{T}$ on a letter $l$ can be applied in different positions of a trinucleotide $x=l_{1} l_{2} l_{3}$ : $\mathscr{T}^{i}, i \in\{1,2,3\}$, is the transition on the position $i$ of $x, \mathscr{T}^{i, j}$, $i, j \in\{1,2,3\}$ with $i<j$, is the transition on the two positions $i$ and $j$ of $x$, and $\mathscr{T}^{1,2,3}$ is the transition on the three positions of $x$.

Example 26. $\mathscr{T}^{1}(A C G)=G C G, \mathscr{T}^{2}(A C G)=A T G, \mathscr{T}^{3}$ $(A C G)=A C A, \mathscr{T}^{1,2}(A C G)=G T G, \mathscr{T}^{1,3}(A C G)=G C A$, $\mathscr{T}^{2,3}(A C G)=A T A$, and $\mathscr{T}^{1,2,3}(A C G)=G T A$.

Definition 27. The transition maps $\mathscr{T}^{i}, \mathscr{T}^{i, j}, \mathscr{T}^{1,2,3}$ on a trinucleotide $x$ are also extended to a trinucleotide code $X$, in a similar way to the genetic maps $\mathscr{C}$ and $\mathscr{P}$.
Definition 28. The transversion I evolution genetic map $\mathscr{V}_{\mathrm{I}}$ : $\mathscr{A}_{4}^{+} \rightarrow \mathscr{A}_{4}^{+}$is defined by

$$
\begin{array}{ll}
\mathscr{V}_{\mathrm{I}}(A)=T, & \mathscr{V}_{\mathrm{I}}(C)=G, \\
\mathscr{V}_{\mathrm{I}}(G)=C, & \mathscr{V}_{\mathrm{I}}(T)=A .
\end{array}
$$

Definition 29. The transversion I map $\mathscr{V}_{\text {I }}$ on a letter $l$ can also be applied in different positions of a trinucleotide $x=l_{1} l_{2} l_{3}$ : $\mathscr{V}_{\mathrm{I}}^{i}, i \in\{1,2,3\}$, is the transversion I on the position $i$ of $x$, $\mathscr{V}_{\mathrm{I}}^{i, j}, i, j \in\{1,2,3\}$ with $i<j$, is the transversion I on the two positions $i$ and $j$ of $x$, and $\mathscr{V}_{\mathrm{I}}^{1,2,3}$ is the transversion I on the three positions of $x$.

Example 30. $\mathscr{V}_{\mathrm{I}}^{1}(A C G)=$ TCG, $\mathscr{V}_{\mathrm{I}}^{2}(A C G)=A G G, \mathscr{V}_{\mathrm{I}}^{3}$ $(A C G)=A C C, \mathscr{V}_{\mathrm{I}}^{1,2}(A C G)=T G G, \mathscr{V}_{\mathrm{I}}^{1,3}(A C G)=T C C$, $\mathscr{V}_{\mathrm{I}}^{2,3}(A C G)=A G C$, and $\mathscr{V}_{\mathrm{I}}^{1,2,3}(A C G)=T G C$.

Definition 31. The transversion I maps $\mathscr{V}_{\mathrm{I}}^{i}, \mathscr{V}_{\mathrm{I}}^{i, j}, \mathscr{V}_{\mathrm{I}}^{1,2,3}$ on a trinucleotide $x$ are also extended to a trinucleotide code $X$, in a similar way to the genetic maps $\mathscr{C}$ and $\mathscr{P}$.

Definition 32. The transversion II evolution genetic map $\mathscr{V}_{\mathrm{II}}$ : $\mathscr{A}_{4}^{+} \rightarrow \mathscr{A}_{4}^{+}$is defined by

$$
\begin{array}{ll}
\mathscr{V}_{\text {II }}(A)=C, & \mathscr{V}_{\text {II }}(C)=A, \\
\mathscr{V}_{\text {II }}(G)=T, & \mathscr{V}_{\text {II }}(T)=G .
\end{array}
$$

Definition 33. The transversion II map $\mathscr{V}_{\text {II }}$ on a letter $l$ can also be applied in different positions of a trinucleotide $x=$ $l_{1} l_{2} l_{3}: \mathscr{V}_{\mathrm{II}}^{i}, i \in\{1,2,3\}$, is the transversion II on the position $i$ of $x, \mathscr{V}_{\mathrm{II}}^{i, j}, i, j \in\{1,2,3\}$ with $i<j$, is the transversion II on the two positions $i$ and $j$ of $x$, and $\mathscr{V}_{\text {II }}^{1,2,3}$ is the transversion II on the three positions of $x$.

Example 34. $\mathscr{V}_{\mathrm{II}}^{1}(A C G)=C C G, \mathscr{V}_{\mathrm{II}}^{2}(A C G)=A A G, \mathscr{V}_{\mathrm{II}}^{3}$ $(A C G)=A C T, \mathscr{V}_{\mathrm{II}}^{1,2}(A C G)=C A G, \mathscr{V}_{\mathrm{II}}^{1,3}(A C G)=C C T$, $\mathscr{V}_{\mathrm{II}}^{2,3}(A C G)=A A T$, and $\mathscr{V}_{\mathrm{II}}^{1,2,3}(A C G)=C A T$.

Definition 35. The transversion II maps $\mathscr{V}_{\text {II }}^{i}, \mathscr{V}_{\text {II }}^{i, j}, \mathscr{V}_{\text {II }}^{1,2,3}$ on a trinucleotide $x$ are also extended to a trinucleotide code $X$, in a similar way to the genetic maps $\mathscr{C}$ and $\mathscr{P}$.

Definition 36. The evolution genetic maps in $l$ trinucleotides of a trinucleotide circular code are defined by $\mathscr{T}(l)$ for transition, $\mathscr{V}_{\mathrm{I}}(l)$ for transversion I, and $\mathscr{V}_{\text {II }}(l)$ for transversion II.

\section{Results}

An evolution genetic map, that is, $\mathscr{T}(l), \mathscr{V}_{\mathrm{I}}(l)$, and $\mathscr{V}_{\mathrm{II}}(l)$, in $l$ trinucleotides of the common trinucleotide circular code $X_{0}$ leads to $S(l)=\left(\begin{array}{c}20 \\ l\end{array}\right)$ trinucleotide codes which are potentially circular. Table 1 gives these numbers $S(l)$.

Based on Proposition 6 allowing to test if a trinucleotide code is circular or not (algorithm not detailed, see, e.g., [2]), computer analyses of a great number of trinucleotide codes allow to identify here new properties with the common trinucleotide circular code $X_{0}$ observed in genes under evolution by transition and transversion. 
TABLE 1: Number $S(l)=\left(\begin{array}{c}20 \\ l\end{array}\right)$ of trinucleotide codes after an evolution genetic map (transition $\mathscr{T}(l)$, transversion I $\mathscr{V}_{\mathrm{I}}(l)$, and transversion II $\left.\mathscr{V}_{\text {II }}(l)\right)$ in $l$ trinucleotides of the common trinucleotide circular code $X_{0}$.

\begin{tabular}{lc}
\hline$l$ & $S(l)$ \\
\hline$\{1,19\}$ & 20 \\
$\{2,18\}$ & 190 \\
$\{3,17\}$ & 1140 \\
$\{4,16\}$ & 4845 \\
$\{5,15\}$ & 15504 \\
$\{6,14\}$ & 38760 \\
$\{7,13\}$ & 77520 \\
$\{8,12\}$ & 125970 \\
$\{9,11\}$ & 167960 \\
10 & 184756 \\
20 & 1 \\
\hline
\end{tabular}

\subsection{Transition Map}

\subsubsection{Transition Map $\mathscr{T}^{i}$}

Result 1 (Table 2). For $l=1, \ldots, 20$

$$
\begin{aligned}
& c\left(\mathscr{T}^{1}(l)\right)=c\left(\mathscr{T}^{3}(l)\right), \\
& \operatorname{mc}\left(\mathscr{T}^{1}(l)\right)=\operatorname{mc}\left(\mathscr{T}^{3}(l)\right), \\
& \operatorname{sc}\left(\mathscr{T}^{1}(l)\right)=\operatorname{sc}\left(\mathscr{T}^{3}(l)\right), \\
& c^{3}\left(\mathscr{T}^{1}(l)\right)=c^{3}\left(\mathscr{T}^{3}(l)\right), \\
& \operatorname{msc}^{3}\left(\mathscr{T}^{1}(l)\right)=\operatorname{msc}^{3}\left(\mathscr{T}^{3}(l)\right) .
\end{aligned}
$$

As expected, the lists of trinucleotide circular codes $C$ associated with $c\left(\mathscr{T}^{1}(l)\right)$ and $c\left(\mathscr{T}^{3}(l)\right)$ are different for $l=$ $1, \ldots, 13$ (not shown). No trinucleotide code is circular after a certain number of transitions $\mathscr{T}^{i}$ in the trinucleotides of the common trinucleotide circular code $X_{0}$. Precisely, for $l=14, \ldots, 20$

$$
c\left(\mathscr{T}^{1}(l)\right)=c\left(\mathscr{T}^{3}(l)\right)=0
$$

and for $l=10, \ldots, 20$

$$
c\left(\mathscr{T}^{2}(l)\right)=0
$$

The transition $\mathscr{T}^{i}$ generates a maximum number of trinucleotide circular codes $C$ for

$$
\begin{array}{r}
\max \left\{c\left(\mathscr{T}^{i}(l)\right), i=1,2,3, l=1, \ldots, 20\right\} \\
=c\left(\mathscr{T}^{1}(7)\right)=c\left(\mathscr{T}^{3}(7)\right)=1436
\end{array}
$$

and a maximum number of $C^{3}$ self-complementary maximum circular codes $\mathrm{MSC}^{3}$ for

$$
\begin{aligned}
\max & \left\{\operatorname{msc}^{3}\left(\mathscr{T}^{i}(l)\right), i=1,2,3, l=1, \ldots, 20\right\} \\
& =\operatorname{msc}^{3}\left(\mathscr{T}^{1}(6)\right)=\operatorname{msc}^{3}\left(\mathscr{T}^{3}(6)\right)=20 .
\end{aligned}
$$

TABle 2: Transition map $\mathscr{T}^{i}(l)$ in $l$ trinucleotides of the common trinucleotide circular code $X_{0}$. Number $c\left(\mathscr{T}^{i}(l)\right)$ of circular codes $C$, number $\operatorname{mc}\left(\mathscr{T}^{i}(l)\right)$ of maximum circular codes $\mathrm{MC}$, number $\operatorname{sc}\left(\mathscr{T}^{i}(l)\right)$ of self-complementary circular codes SC, number $c^{3}\left(\mathscr{T}^{i}(l)\right)$ of circular codes $C^{3}$, and number $\operatorname{msc}^{3}\left(\mathscr{T}^{i}(l)\right)$ of $C^{3}$ selfcomplementary maximum circular codes $\mathrm{MSC}^{3}$.

\begin{tabular}{lcccccccccc}
\hline & \multicolumn{4}{c}{$\mathscr{T}^{1}(l)$ or $\mathscr{T}^{3}(l)$} & \multicolumn{5}{c}{$\mathscr{T}^{2}(l)$} \\
& $c$ & $\mathrm{mc}$ & $\mathrm{sc}$ & $c^{3}$ & $\mathrm{msc}^{3}$ & $c$ & $\mathrm{mc}$ & $\mathrm{sc}$ & $c^{3}$ & $\mathrm{msc}^{3}$ \\
\hline 1 & 12 & 0 & 0 & 12 & 0 & 8 & 0 & 0 & 8 & 0 \\
2 & 68 & 6 & 10 & 68 & 6 & 28 & 4 & 8 & 28 & 4 \\
3 & 240 & 0 & 0 & 240 & 0 & 60 & 0 & 0 & 56 & 0 \\
4 & 587 & 15 & 33 & 586 & 15 & 92 & 6 & 18 & 74 & 6 \\
5 & 1049 & 0 & 0 & 1040 & 0 & 108 & 0 & 0 & 62 & 0 \\
6 & 1408 & 20 & 48 & 1372 & 20 & 96 & 4 & 8 & 32 & 4 \\
7 & 1436 & 0 & 0 & 1352 & 0 & 60 & 0 & 0 & 8 & 0 \\
8 & 1111 & 15 & 33 & 985 & 15 & 23 & 1 & 1 & 1 & 1 \\
9 & 642 & 0 & 0 & 516 & 0 & 4 & 0 & 0 & 0 & 0 \\
10 & 268 & 6 & 10 & 184 & 6 & 0 & 0 & 0 & 0 & 0 \\
11 & 76 & 0 & 0 & 40 & 0 & 0 & 0 & 0 & 0 & 0 \\
12 & 13 & 1 & 1 & 4 & 1 & 0 & 0 & 0 & 0 & 0 \\
13 & 1 & 0 & 0 & 0 & 0 & 0 & 0 & 0 & 0 & 0 \\
$\{14, \ldots, 20\}$ & 0 & 0 & 0 & 0 & 0 & 0 & 0 & 0 & 0 & 0 \\
\hline
\end{tabular}

\subsubsection{Transition Map $\mathscr{T}^{i, j}$}

Result 2 (Table 3). For $l=1, \ldots, 20$

$$
\begin{aligned}
& c\left(\mathscr{T}^{1,2}(l)\right)=c\left(\mathscr{T}^{2,3}(l)\right), \\
& \operatorname{mc}\left(\mathscr{T}^{1,2}(l)\right)=\operatorname{mc}\left(\mathscr{T}^{2,3}(l)\right), \\
& \operatorname{sc}\left(\mathscr{T}^{1,2}(l)\right)=\operatorname{sc}\left(\mathscr{T}^{2,3}(l)\right), \\
& c^{3}\left(\mathscr{T}^{1,2}(l)\right)=c^{3}\left(\mathscr{T}^{2,3}(l)\right), \\
& \operatorname{msc}^{3}\left(\mathscr{T}^{1,2}(l)\right)=\operatorname{msc}^{3}\left(\mathscr{T}^{2,3}(l)\right) .
\end{aligned}
$$

The lists of trinucleotide circular codes $C$ associated with $c\left(\mathscr{T}^{1,2}(l)\right)$ and $c\left(\mathscr{T}^{2,3}(l)\right)$ are different for $l=1, \ldots, 14$ (not shown). No trinucleotide code is circular after a certain number of transitions $\mathscr{T}^{i, j}$ in the trinucleotides of the common trinucleotide circular code $X_{0}$. Precisely, for $l=15, \ldots, 20$

$$
c\left(\mathscr{T}^{1,2}(l)\right)=c\left(\mathscr{T}^{2,3}(l)\right)=0
$$

and for $l=12, \ldots, 20$

$$
c\left(\mathscr{T}^{1,3}(l)\right)=0
$$

The transition $\mathscr{T}^{i, j}$ generates a maximum number of trinucleotide circular codes $C$ for

$$
\begin{aligned}
\max & \left\{c\left(\mathscr{T}^{i, j}(l)\right), i, j=1,2,3, i<j, l=1, \ldots, 20\right\} \\
& =c\left(\mathscr{T}^{1,3}(6)\right)=598
\end{aligned}
$$


TABLE 3: Transition map $\mathscr{T}^{i, j}(l)$ in $l$ trinucleotides of the common trinucleotide circular code $X_{0}$. Number $c\left(\mathscr{T}^{i, j}(l)\right)$ of circular codes $C$, number $\operatorname{mc}\left(\mathscr{T}^{i, j}(l)\right)$ of maximum circular codes MC, number sc $\left(\mathscr{T}^{i, j}(l)\right)$ of self-complementary circular codes SC, number $c^{3}\left(\mathscr{T}^{i, j}(l)\right)$ of circular codes $C^{3}$, and number $\operatorname{msc}^{3}\left(\mathscr{T}^{i, j}(l)\right)$ of $C^{3}$ self-complementary maximum circular codes MSC ${ }^{3}$.

\begin{tabular}{|c|c|c|c|c|c|c|c|c|c|c|}
\hline \multirow{2}{*}{$l$} & \multicolumn{5}{|c|}{$\mathscr{T}^{1,2}(l)$ or $\mathscr{T}^{2,3}(l)$} & \multicolumn{5}{|c|}{$\mathscr{T}^{1,3}(l)$} \\
\hline & $c$ & $\mathrm{mc}$ & sc & $c^{3}$ & $\mathrm{msc}^{3}$ & $c$ & $\mathrm{mc}$ & sc & $c^{3}$ & $\mathrm{msc}^{3}$ \\
\hline 1 & 9 & 1 & 0 & 9 & 0 & 10 & 2 & 0 & 10 & 0 \\
\hline 2 & 37 & 4 & 6 & 36 & 4 & 49 & 5 & 9 & 45 & 5 \\
\hline 3 & 91 & 4 & 0 & 85 & 0 & 152 & 8 & 0 & 122 & 0 \\
\hline 4 & 148 & 6 & 11 & 130 & 6 & 325 & 10 & 27 & 223 & 10 \\
\hline 5 & 166 & 6 & 0 & 133 & 0 & 508 & 12 & 0 & 292 & 0 \\
\hline 6 & 129 & 4 & 6 & 90 & 4 & 598 & 10 & 30 & 286 & 10 \\
\hline 7 & 67 & 4 & 0 & 38 & 0 & 532 & 8 & 0 & 214 & 0 \\
\hline 8 & 21 & 1 & 1 & 9 & 1 & 353 & 5 & 11 & 121 & 5 \\
\hline 9 & 4 & 1 & 0 & 1 & 0 & 162 & 2 & 0 & 44 & 0 \\
\hline 10 & 3 & 0 & 0 & 0 & 0 & 42 & 1 & 2 & 10 & 1 \\
\hline 11 & 4 & 0 & 0 & 0 & 0 & 4 & 0 & 0 & 0 & 0 \\
\hline 12 & 3 & 0 & 0 & 0 & 0 & 0 & 0 & 0 & 0 & 0 \\
\hline 13 & 4 & 0 & 0 & 0 & 0 & 0 & 0 & 0 & 0 & 0 \\
\hline 14 & 1 & 0 & 0 & 1 & 0 & 0 & 0 & 0 & 0 & 0 \\
\hline$\{15, \ldots, 20\}$ & 0 & 0 & 0 & 0 & 0 & 0 & 0 & 0 & 0 & 0 \\
\hline
\end{tabular}

TABLE 4: Transition map $\mathscr{T}^{1,2,3}(l)$ in $l$ trinucleotides of the common trinucleotide circular code $X_{0}$. Number $c\left(\mathscr{T}^{1,2,3}(l)\right)$ of circular codes $C$, number $\operatorname{mc}\left(\mathscr{T}^{1,2,3}(l)\right)$ of maximum circular codes MC, number $\operatorname{sc}\left(\mathscr{T}^{1,2,3}(l)\right)$ of self-complementary circular codes SC, number $c^{3}\left(\mathscr{T}^{1,2,3}(l)\right)$ of circular codes $C^{3}$, and number $\operatorname{msc}^{3}\left(\mathscr{T}^{1,2,3}(l)\right)$ of $C^{3}$ self-complementary maximum circular codes $\mathrm{MSC}^{3}$.

\begin{tabular}{lccccc}
\hline$l$ & \multicolumn{4}{c}{$\mathscr{T}^{1,2,3}(l)$} & \\
& $c$ & $\mathrm{mc}$ & $\mathrm{sc}$ & $c^{3}$ & $\mathrm{msc}^{3}$ \\
\hline 1,19$\}$ & 8 & 0 & 0 & 8 & 0 \\
$\{2,18\}$ & 28 & 4 & 8 & 28 & 4 \\
$\{3,17\}$ & 56 & 0 & 0 & 56 & 0 \\
$\{4,16\}$ & 71 & 7 & 19 & 70 & 6 \\
$\{5,15\}$ & 64 & 0 & 0 & 56 & 0 \\
$\{6,14\}$ & 56 & 8 & 16 & 28 & 4 \\
$\{7,13\}$ & 64 & 0 & 0 & 8 & 0 \\
$\{8,12\}$ & 72 & 8 & 20 & 1 & 1 \\
$\{9,11\}$ & 64 & 0 & 0 & 0 & 0 \\
10 & 56 & 8 & 16 & 0 & 0 \\
20 & 1 & 1 & 1 & 1 & 1 \\
\hline
\end{tabular}

and a maximum number of $C^{3}$ self-complementary maximum circular codes $\mathrm{MSC}^{3}$ for

$$
\begin{aligned}
\max & \left\{\operatorname{msc}^{3}\left(\mathscr{T}^{i, j}(l)\right), i, j=1,2,3, i<j, l=1, \ldots, 20\right\} \\
& =\operatorname{msc}^{3}\left(\mathscr{T}^{1,3}(4)\right)=\operatorname{msc}^{3}\left(\mathscr{T}^{1,3}(6)\right)=10 .
\end{aligned}
$$

The numbers $c^{3}\left(\mathscr{T}^{1,2}(l)\right)=c^{3}\left(\mathscr{T}^{2,3}(l)\right)$ of circular codes $C^{3}$ have a particular growth function

$$
c^{3}\left(\mathscr{T}^{1,2}(14)\right)=c^{3}\left(\mathscr{T}^{2,3}(14)\right)=1,
$$

$$
c^{3}\left(\mathscr{T}^{1,2}(l)\right)=c^{3}\left(\mathscr{T}^{2,3}(l)\right)=0 \quad \text { for } l=10, \ldots, 13 .
$$

\subsubsection{Transition Map $\mathscr{T}^{1,2,3}$}

Result 3 (Table 4). The transition $\mathscr{T}^{1,2,3}$ always generates trinucleotide circular codes. Indeed, for $l=1, \ldots, 20$

$$
c\left(\mathscr{T}^{1,2,3}(l)\right)>0 .
$$

The lists of trinucleotide circular codes $C$ associated with $c\left(\mathscr{T}^{1,2,3}(l)\right)$ and $c\left(\mathscr{T}^{1,2,3}(20-l)\right)$ are different for $l=1, \ldots, 9$ (not shown). The transition $\mathscr{T}^{1,2,3}$ generates a maximum number of trinucleotide circular codes $C$ for

$$
\begin{aligned}
\max & \left\{c\left(\mathscr{T}^{1,2,3}(l)\right), l=1, \ldots, 20\right\} \\
& =c\left(\mathscr{T}^{1,2,3}(8)\right)=c\left(\mathscr{T}^{1,2,3}(12)\right)=72
\end{aligned}
$$

and a maximum number of $C^{3}$ self-complementary maximum circular codes MSC ${ }^{3}$ for

$$
\begin{aligned}
\max & \left\{\operatorname{msc}^{3}\left(\mathscr{T}^{1,2,3}(l)\right), l=1, \ldots, 20\right\} \\
& =\operatorname{msc}^{3}\left(\mathscr{T}^{1,2,3}(4)\right)=\operatorname{msc}^{3}\left(\mathscr{T}^{1,2,3}(16)\right)=6 .
\end{aligned}
$$

\subsection{Transversion I Map}

\subsubsection{Transversion I Map $\mathscr{V}_{I}^{i}$}

Result 4 (Table 5). For $l=1, \ldots, 20$

$$
\begin{aligned}
& c\left(\mathscr{V}_{\mathrm{I}}^{1}(l)\right)=c\left(\mathscr{V}_{\mathrm{I}}^{3}(l)\right), \\
& \operatorname{mc}\left(\mathscr{V}_{\mathrm{I}}^{1}(l)\right)=\operatorname{mc}\left(\mathscr{V}_{\mathrm{I}}^{3}(l)\right), \\
& \operatorname{sc}\left(\mathscr{V}_{\mathrm{I}}^{1}(l)\right)=\operatorname{sc}\left(\mathscr{V}_{\mathrm{I}}^{3}(l)\right),
\end{aligned}
$$




$$
\begin{aligned}
& c^{3}\left(\mathscr{V}_{\mathrm{I}}^{1}(l)\right)=c^{3}\left(\mathscr{V}_{\mathrm{I}}^{3}(l)\right), \\
& \operatorname{msc}^{3}\left(\mathscr{V}_{\mathrm{I}}^{1}(l)\right)=\operatorname{msc}^{3}\left(\mathscr{V}_{\mathrm{I}}^{3}(l)\right) .
\end{aligned}
$$

The lists of trinucleotide circular codes $C$ associated with $c\left(\mathscr{V}_{\mathrm{I}}^{1}(l)\right)$ and $c\left(\mathscr{V}_{\mathrm{I}}^{3}(l)\right)$ are different for $l=1, \ldots, 9$ (not shown). No trinucleotide code is circular after a certain number of transversions $\mathrm{I}_{\mathrm{V}_{\mathrm{I}}^{i}}^{i}$ in the trinucleotides of the common trinucleotide circular code $X_{0}$. Precisely, for $l=$ $10, \ldots, 20$

$$
c\left(\mathscr{V}_{\mathrm{I}}^{1}(l)\right)=c\left(\mathscr{V}_{\mathrm{I}}^{3}(l)\right)=0
$$

and for $l=18,19,20$

$$
c\left(\mathscr{V}_{\mathrm{I}}^{2}(l)\right)=0
$$

The transversion $\mathrm{I}_{\mathscr{V}_{\mathrm{I}}^{i}}^{i}$ generates a maximum number of trinucleotide circular codes $C$ for

$$
\begin{aligned}
\max & \left\{c\left(\mathscr{V}_{\mathrm{I}}^{i}(l)\right), i=1,2,3, l=1, \ldots, 20\right\} \\
& =c\left(\mathscr{V}_{\mathrm{I}}^{2}(9)\right)=24310
\end{aligned}
$$

and a maximum number of $C^{3}$ self-complementary maximum circular codes $\mathrm{MSC}^{3}$ for

$$
\begin{aligned}
\max & \left\{\operatorname{msc}^{3}\left(\mathscr{V}_{\mathrm{I}}^{i}(l)\right), i=1,2,3, l=1, \ldots, 20\right\} \\
& =\operatorname{msc}^{3}\left(\mathscr{V}_{\mathrm{I}}^{2}(8)\right)=70 .
\end{aligned}
$$

A remarkable code property only found with transversion I $\mathscr{V}_{\mathrm{I}}^{2}$ is, for $l=1, \ldots, 20$,

$$
c\left(\mathscr{V}_{\mathrm{I}}^{2}(l)\right)=c^{3}\left(\mathscr{V}_{\mathrm{I}}^{2}(l)\right)
$$

and furthermore, after a detailed computer analysis, the lists of trinucleotide circular codes $C$ and $C^{3}$ associated with $c\left(\mathscr{V}_{\mathrm{I}}^{2}(l)\right)$ and $c^{3}\left(\mathscr{V}_{\mathrm{I}}^{2}(l)\right)$, respectively, are identical for $l=$ $1, \ldots, 17$.

\subsubsection{Transversion I Map $\mathscr{V}_{I}^{i, j}$}

Result 5 (Table 6). For $l=1, \ldots, 20$

$$
\begin{aligned}
& c\left(\mathscr{V}_{\mathrm{I}}^{1,2}(l)\right)=c\left(\mathscr{V}_{\mathrm{I}}^{2,3}(l)\right), \\
& \operatorname{mc}\left(\mathscr{V}_{\mathrm{I}}^{1,2}(l)\right)=\operatorname{mc}\left(\mathscr{V}_{\mathrm{I}}^{2,3}(l)\right), \\
& \operatorname{sc}\left(\mathscr{V}_{\mathrm{I}}^{1,2}(l)\right)=\operatorname{sc}\left(\mathscr{V}_{\mathrm{I}}^{2,3}(l)\right), \\
& c^{3}\left(\mathscr{V}_{\mathrm{I}}^{1,2}(l)\right)=c^{3}\left(\mathscr{V}_{\mathrm{I}}^{2,3}(l)\right), \\
& \operatorname{msc}^{3}\left(\mathscr{V}_{\mathrm{I}}^{1,2}(l)\right)=\operatorname{msc}^{3}\left(\mathscr{V}_{\mathrm{I}}^{2,3}(l)\right) .
\end{aligned}
$$

The lists of trinucleotide circular codes $C$ associated with $c\left(\mathscr{V}_{\mathrm{I}}^{1,2}(l)\right)$ and $c\left(\mathscr{V}_{\mathrm{I}}^{2,3}(l)\right)$ are different for $l=1, \ldots, 12$ (not shown). No trinucleotide code is circular after a certain number of transversions $\mathrm{I} \mathscr{V}_{\mathrm{I}}^{i, j}$ in the trinucleotides of the common trinucleotide circular code $X_{0}$. Precisely, for $l=$ $13, \ldots, 20$

$$
c\left(\mathscr{V}_{\mathrm{I}}^{1,2}(l)\right)=c\left(\mathscr{V}_{\mathrm{I}}^{2,3}(l)\right)=0
$$

and for $l=19,20$

$$
c\left(\mathscr{V}_{\mathrm{I}}^{1,3}(l)\right)=0
$$

The transversion $\mathrm{I} \mathscr{V}_{\mathrm{I}}^{i, j}$ generates a maximum number of trinucleotide circular codes $C$ for

$$
\begin{gathered}
\max \left\{c\left(\mathscr{V}_{\mathrm{I}}^{i, j}(l)\right), i, j=1,2,3, i<j, l=1, \ldots, 20\right\} \\
=c\left(\mathscr{V}_{\mathrm{I}}^{1,2}(6)\right)=c\left(\mathscr{V}_{\mathrm{I}}^{2,3}(6)\right)=630
\end{gathered}
$$

and a maximum number of $C^{3}$ self-complementary maximum circular codes $\mathrm{MSC}^{3}$ for

$$
\begin{aligned}
\max & \left\{\operatorname{msc}^{3}\left(\mathscr{V}_{\mathrm{I}}^{i, j}(l)\right), i, j=1,2,3, i<j, l=1, \ldots, 20\right\} \\
& =\operatorname{msc}^{3}\left(\mathscr{V}_{\mathrm{I}}^{1,2}(4)\right)=\operatorname{msc}^{3}\left(\mathscr{V}_{\mathrm{I}}^{2,3}(4)\right)=6 .
\end{aligned}
$$

The numbers $\operatorname{sc}\left(\mathscr{V}_{\mathrm{I}}^{1,3}(l)\right)$ of self-complementary circular codes SC have a particular growth function

$$
\begin{gathered}
\operatorname{sc}\left(\mathscr{V}_{\mathrm{I}}^{1,3}(l)\right)=1 \quad \text { for } l=12,14,16,18, \\
\operatorname{sc}\left(\mathscr{V}_{\mathrm{I}}^{1,3}(l)\right)=0 \quad \text { for } l=8,10 .
\end{gathered}
$$

The numbers $c^{3}\left(\mathscr{V}_{\mathrm{I}}^{1,3}(l)\right)$ of circular codes $C^{3}$ have a particular growth function

$$
\begin{gathered}
c^{3}\left(\mathscr{V}_{\mathrm{I}}^{1,3}(l)\right)=1 \quad \text { for } l=16,18, \\
c^{3}\left(\mathscr{V}_{\mathrm{I}}^{1,3}(17)\right)=2, \\
c^{3}\left(\mathscr{V}_{\mathrm{I}}^{1,3}(l)\right)=0 \quad \text { for } l=7, \ldots, 15 .
\end{gathered}
$$

\subsubsection{Transversion I Map $\mathscr{V}_{I}^{1,2,3}$}

Result 6 (Table 7). The transversion I $\mathscr{V}_{\mathrm{I}}^{1,2,3}$ always generates trinucleotide circular codes. Indeed, for $l=1, \ldots, 20$

$$
c\left(\mathscr{V}_{\mathrm{I}}^{1,2,3}(l)\right)>0 \text {. }
$$

The lists of trinucleotide circular codes $C$ associated with $c\left(\mathscr{V}_{\mathrm{I}}^{1,2,3}(l)\right)$ and $c\left(\mathscr{V}_{\mathrm{I}}^{1,2,3}(20-l)\right)$ are different for $l=1, \ldots, 9$ (not shown). The transversion $\mathrm{I} \mathscr{V}_{\mathrm{I}}^{1,2,3}$ generates a maximum number of trinucleotide circular codes $C$ for

$$
\max \left\{c\left(\mathscr{V}_{\mathrm{I}}^{1,2,3}(l)\right), l=1, \ldots, 20\right\}=c\left(\mathscr{V}_{\mathrm{I}}^{1,2,3}(10)\right)=66
$$

and a maximum number of $C^{3}$ self-complementary maximum circular codes $\mathrm{MSC}^{3}$ for

$$
\begin{aligned}
\max & \left\{\operatorname{msc}^{3}\left(\mathscr{V}_{\mathrm{I}}^{1,2,3}(l)\right), l=1, \ldots, 20\right\} \\
& =\operatorname{msc}^{3}\left(\mathscr{V}_{\mathrm{I}}^{1,2,3}(4)\right)=\operatorname{msc}^{3}\left(\mathscr{V}_{\mathrm{I}}^{1,2,3}(16)\right)=9 .
\end{aligned}
$$


TABLE 5: Transversion I map $\mathscr{V}_{\mathrm{I}}^{i}(l)$ in $l$ trinucleotides of the common trinucleotide circular code $X_{0}$. Number $c\left(\mathscr{V}_{\mathrm{I}}^{i}(l)\right)$ of circular codes $C$, number $\operatorname{mc}\left(\mathscr{V}_{\mathrm{I}}^{i}(l)\right)$ of maximum circular codes $\mathrm{MC}$, number sc $\left(\mathscr{V}_{\mathrm{I}}^{i}(l)\right)$ of self-complementary circular codes $\mathrm{SC}$, number $c^{3}\left(\mathscr{V}_{\mathrm{I}}^{i}(l)\right)$ of $\operatorname{circular}$ codes $C^{3}$, and number $\mathrm{msc}^{3}\left(\mathscr{V}_{\mathrm{I}}^{i}(l)\right)$ of $C^{3}$ self-complementary maximum circular codes $\mathrm{MSC}^{3}$.

\begin{tabular}{|c|c|c|c|c|c|c|c|c|c|c|}
\hline \multirow{2}{*}{$l$} & \multicolumn{5}{|c|}{$\mathscr{V}_{\mathrm{I}}^{1}(l)$ or $\mathscr{V}_{\mathrm{I}}^{3}(l)$} & \multicolumn{5}{|c|}{$\mathscr{V}_{\mathrm{I}}^{2}(l)$} \\
\hline & $c$ & $\mathrm{mc}$ & $\mathrm{sc}$ & $c^{3}$ & $\mathrm{msc}^{3}$ & $c$ & $\mathrm{mc}$ & $\mathrm{sc}$ & $c^{3}$ & $\mathrm{msc}^{3}$ \\
\hline 1 & 8 & 0 & 0 & 8 & 0 & 16 & 0 & 0 & 16 & 0 \\
\hline 2 & 31 & 4 & 5 & 30 & 4 & 122 & 8 & 12 & 122 & 8 \\
\hline 3 & 76 & 0 & 0 & 71 & 0 & 590 & 0 & 0 & 590 & 0 \\
\hline 4 & 130 & 6 & 8 & 114 & 6 & 2030 & 28 & 56 & 2030 & 28 \\
\hline 5 & 160 & 0 & 0 & 125 & 0 & 5278 & 0 & 0 & 5278 & 0 \\
\hline 6 & 142 & 4 & 5 & 92 & 4 & 10738 & 56 & 132 & 10738 & 56 \\
\hline 7 & 87 & 0 & 0 & 42 & 0 & 17446 & 0 & 0 & 17446 & 0 \\
\hline 8 & 32 & 1 & 1 & 10 & 1 & 22880 & 70 & 174 & 22880 & 70 \\
\hline 9 & 5 & 0 & 0 & 1 & 0 & 24310 & 0 & 0 & 24310 & 0 \\
\hline 10 & 0 & 0 & 0 & 0 & 0 & 20878 & 56 & 132 & 20878 & 56 \\
\hline 11 & 0 & 0 & 0 & 0 & 0 & 14378 & 0 & 0 & 14378 & 0 \\
\hline 12 & 0 & 0 & 0 & 0 & 0 & 7826 & 28 & 56 & 7826 & 28 \\
\hline 13 & 0 & 0 & 0 & 0 & 0 & 3290 & 0 & 0 & 3290 & 0 \\
\hline 14 & 0 & 0 & 0 & 0 & 0 & 1030 & 8 & 12 & 1030 & 8 \\
\hline 15 & 0 & 0 & 0 & 0 & 0 & 226 & 0 & 0 & 226 & 0 \\
\hline 16 & 0 & 0 & 0 & 0 & 0 & 31 & 1 & 1 & 31 & 1 \\
\hline 17 & 0 & 0 & 0 & 0 & 0 & 2 & 0 & 0 & 2 & 0 \\
\hline$\{18, \ldots, 20\}$ & 0 & 0 & 0 & 0 & 0 & 0 & 0 & 0 & 0 & 0 \\
\hline
\end{tabular}

TABLE 6: Transversion I map $\mathscr{V}_{\mathrm{I}}^{i, j}(l)$ in $l$ trinucleotides of the common trinucleotide circular code $X_{0}$. Number $c\left(\mathscr{V}_{\mathrm{I}}^{i, j}(l)\right)$ of circular codes $C$, number $\operatorname{mc}\left(\mathscr{V}_{\mathrm{I}}^{i, j}(l)\right)$ of maximum circular codes MC, number $\operatorname{sc}\left(\mathscr{V}_{\mathrm{I}}^{i, j}(l)\right)$ of self-complementary circular codes $\mathrm{SC}$, number $c^{3}\left(\mathscr{V}_{\mathrm{I}}^{i, j}(l)\right)$ of circular codes $C^{3}$, and number $\operatorname{msc}^{3}\left(\mathscr{V}_{\mathrm{I}}^{i, j}(l)\right)$ of $C^{3}$ self-complementary maximum circular codes MSC ${ }^{3}$.

\begin{tabular}{|c|c|c|c|c|c|c|c|c|c|c|}
\hline \multirow{2}{*}{$l$} & \multicolumn{5}{|c|}{$\mathscr{V}_{\mathrm{I}}^{1,2}(l)$ or $\mathscr{V}_{\mathrm{I}}^{2,3}(l)$} & \multicolumn{5}{|c|}{$\mathscr{V}_{\mathrm{I}}^{1,3}(l)$} \\
\hline & $c$ & $\mathrm{mc}$ & $\mathrm{sc}$ & $c^{3}$ & $\mathrm{msc}^{3}$ & $c$ & $\mathrm{mc}$ & $\mathrm{sc}$ & $c^{3}$ & $\mathrm{msc}^{3}$ \\
\hline 1 & 10 & 2 & 0 & 9 & 0 & 6 & 2 & 0 & 4 & 0 \\
\hline 2 & 48 & 5 & 5 & 38 & 4 & 15 & 3 & 5 & 7 & 3 \\
\hline 3 & 147 & 8 & 0 & 102 & 0 & 20 & 4 & 0 & 8 & 0 \\
\hline 4 & 319 & 10 & 8 & 191 & 6 & 15 & 3 & 5 & 7 & 3 \\
\hline 5 & 514 & 12 & 0 & 265 & 0 & 8 & 4 & 0 & 4 & 0 \\
\hline 6 & 630 & 10 & 5 & 282 & 4 & 11 & 3 & 1 & 1 & 1 \\
\hline 7 & 595 & 8 & 0 & 240 & 0 & 22 & 4 & 0 & 0 & 0 \\
\hline 8 & 435 & 5 & 1 & 162 & 1 & 32 & 4 & 0 & 0 & 0 \\
\hline 9 & 245 & 2 & 0 & 88 & 0 & 38 & 2 & 0 & 0 & 0 \\
\hline 10 & 103 & 1 & 0 & 34 & 0 & 38 & 2 & 0 & 0 & 0 \\
\hline 11 & 29 & 0 & 0 & 8 & 0 & 34 & 0 & 0 & 0 & 0 \\
\hline 12 & 4 & 0 & 0 & 1 & 0 & 39 & 0 & 1 & 0 & 0 \\
\hline 13 & 0 & 0 & 0 & 0 & 0 & 56 & 0 & 0 & 0 & 0 \\
\hline 14 & 0 & 0 & 0 & 0 & 0 & 65 & 0 & 1 & 0 & 0 \\
\hline 15 & 0 & 0 & 0 & 0 & 0 & 52 & 0 & 0 & 0 & 0 \\
\hline 16 & 0 & 0 & 0 & 0 & 0 & 27 & 0 & 1 & 1 & 0 \\
\hline 17 & 0 & 0 & 0 & 0 & 0 & 8 & 0 & 0 & 2 & 0 \\
\hline 18 & 0 & 0 & 0 & 0 & 0 & 1 & 0 & 1 & 1 & 0 \\
\hline$\{19,20\}$ & 0 & 0 & 0 & 0 & 0 & 0 & 0 & 0 & 0 & 0 \\
\hline
\end{tabular}


TABLE 7: Transversion I map $\mathscr{V}_{\mathrm{I}}^{1,2,3}(l)$ in $l$ trinucleotides of the common trinucleotide circular code $X_{0}$. Number $c\left(\mathscr{V}_{\mathrm{I}}^{1,2,3}(l)\right)$ of circular codes $C$, number $\operatorname{mc}\left(\mathscr{V}_{\mathrm{I}}^{1,2,3}(l)\right)$ of maximum circular codes MC, number $\operatorname{sc}\left(\mathscr{V}_{\mathrm{I}}^{1,2,3}(l)\right)$ of self-complementary circular codes SC, number $c^{3}\left(\mathscr{V}_{\mathrm{I}}^{1,2,3}(l)\right)$ of circular codes $C^{3}$, and number $\operatorname{msc}^{3}\left(\mathscr{V}_{\mathrm{I}}^{1,2,3}(l)\right)$ of $C^{3}$ self-complementary maximum circular codes $\mathrm{MSC}^{3}$.

\begin{tabular}{lccccc}
\hline$l$ & \multicolumn{4}{c}{$\mathscr{V}_{\mathrm{I}}^{1,2,3}(l)$} & \\
& $c$ & $\mathrm{mc}$ & $\mathrm{sc}$ & $c^{3}$ & $\mathrm{msc}^{3}$ \\
\hline$\{1,19\}$ & 6 & 0 & 0 & 6 & 0 \\
$\{2,18\}$ & 17 & 5 & 7 & 17 & 5 \\
$\{3,17\}$ & 32 & 0 & 0 & 32 & 0 \\
$\{4,16\}$ & 47 & 10 & 17 & 45 & 9 \\
$\{5,15\}$ & 54 & 0 & 0 & 46 & 0 \\
$\{6,14\}$ & 51 & 12 & 19 & 31 & 7 \\
$\{7,13\}$ & 48 & 0 & 0 & 12 & 0 \\
$\{8,12\}$ & 53 & 13 & 11 & 2 & 2 \\
$\{9,11\}$ & 62 & 0 & 0 & 0 & 0 \\
10 & 66 & 14 & 6 & 0 & 0 \\
20 & 1 & 1 & 1 & 1 & 1 \\
\hline
\end{tabular}

TABLE 8: Transversion II map $\mathscr{V}_{\mathrm{II}}^{i}(l)$ in $l$ trinucleotides of the common trinucleotide circular code $X_{0}$. Number $c\left(\mathscr{V}_{\mathrm{II}}^{i}(l)\right)$ of circular codes $C$, number $\operatorname{mc}\left(\mathscr{V}_{\mathrm{II}}^{i}(l)\right)$ of maximum circular codes $\mathrm{MC}$, number $\mathrm{sc}\left(\mathscr{V}_{\mathrm{II}}^{i}(l)\right)$ of self-complementary circular codes SC, number $c^{3}\left(\mathscr{V}_{\mathrm{II}}^{i}(l)\right)$ of circular codes $C^{3}$, and number $\operatorname{msc}^{3}\left(\mathscr{V}_{\mathrm{II}}^{i}(l)\right)$ of $C^{3}$ self-complementary maximum circular codes MSC $^{3}$.

\begin{tabular}{lcccccccccc}
\hline$l$ & \multicolumn{4}{c}{$\mathscr{V}_{\mathrm{II}}^{1}(l)$ or $\mathscr{V}_{\mathrm{II}}^{3}(l)$} & \multicolumn{5}{c}{$\mathscr{V}_{\mathrm{II}}^{2}(l)$} \\
& $c$ & $\mathrm{mc}$ & $\mathrm{sc}$ & $c^{3}$ & $\mathrm{msc}^{3}$ & $c$ & $\mathrm{mc}$ & $\mathrm{sc}$ & $c^{3}$ & $\mathrm{msc}^{3}$ \\
\hline 1 & 6 & 0 & 0 & 6 & 0 & 8 & 0 & 0 & 8 & 0 \\
2 & 17 & 3 & 4 & 17 & 3 & 30 & 4 & 8 & 28 & 4 \\
3 & 30 & 0 & 0 & 29 & 0 & 74 & 0 & 0 & 58 & 0 \\
4 & 35 & 3 & 4 & 31 & 3 & 132 & 6 & 18 & 82 & 6 \\
5 & 28 & 0 & 0 & 20 & 0 & 176 & 0 & 0 & 76 & 0 \\
6 & 16 & 1 & 1 & 7 & 1 & 170 & 4 & 8 & 44 & 4 \\
7 & 6 & 0 & 0 & 1 & 0 & 120 & 0 & 0 & 12 & 0 \\
8 & 1 & 0 & 0 & 0 & 0 & 61 & 1 & 1 & 1 & 1 \\
9 & 0 & 0 & 0 & 0 & 0 & 26 & 0 & 0 & 0 & 0 \\
10 & 0 & 0 & 0 & 0 & 0 & 10 & 0 & 0 & 0 & 0 \\
11 & 0 & 0 & 0 & 0 & 0 & 2 & 0 & 0 & 0 & 0 \\
$\{12, \ldots, 20\}$ & 0 & 0 & 0 & 0 & 0 & 0 & 0 & 0 & 0 & 0 \\
\hline
\end{tabular}

\subsection{Transversion II Map}

\subsubsection{Transversion II Map $\mathscr{V}_{I I}^{i}$}

Result 7 (Table 8). For $l=1, \ldots, 20$

$$
\begin{aligned}
& c\left(\mathscr{V}_{\mathrm{II}}^{1}(l)\right)=c\left(\mathscr{V}_{\mathrm{II}}^{3}(l)\right), \\
& \operatorname{mc}\left(\mathscr{V}_{\mathrm{II}}^{1}(l)\right)=\operatorname{mc}\left(\mathscr{V}_{\mathrm{II}}^{3}(l)\right), \\
& \operatorname{sc}\left(\mathscr{V}_{\mathrm{II}}^{1}(l)\right)=\operatorname{sc}\left(\mathscr{V}_{\mathrm{II}}^{3}(l)\right),
\end{aligned}
$$

$$
\begin{aligned}
c^{3}\left(\mathscr{V}_{\mathrm{II}}^{1}(l)\right) & =c^{3}\left(\mathscr{V}_{\mathrm{II}}^{3}(l)\right), \\
\operatorname{msc}^{3}\left(\mathscr{V}_{\mathrm{II}}^{1}(l)\right) & =\operatorname{msc}^{3}\left(\mathscr{V}_{\mathrm{II}}^{3}(l)\right) .
\end{aligned}
$$

The lists of trinucleotide circular codes $C$ associated with $c\left(\mathscr{V}_{\mathrm{II}}^{1}(l)\right)$ and $c\left(\mathscr{V}_{\mathrm{II}}^{3}(l)\right)$ are different for $l=1, \ldots, 8$ (not shown). No trinucleotide code is circular after a certain number of transversions II $\mathscr{V}_{\mathrm{II}}^{i}$ in the trinucleotides of the common trinucleotide circular code $X_{0}$. Precisely, for $l=$ $9, \ldots, 20$

$$
c\left(\mathscr{V}_{\mathrm{II}}^{1}(l)\right)=c\left(\mathscr{V}_{\mathrm{II}}^{3}(l)\right)=0
$$

and for $l=12, \ldots, 20$

$$
c\left(\mathscr{V}_{\mathrm{II}}^{2}(l)\right)=0
$$

The transversion II $\mathscr{V}_{\text {II }}^{i}$ generates a maximum number of trinucleotide circular codes $C$ for

$$
\begin{aligned}
\max & \left\{c\left(\mathscr{V}_{\mathrm{II}}^{i}(l)\right), i=1,2,3, l=1, \ldots, 20\right\} \\
& =c\left(\mathscr{V}_{\mathrm{II}}^{2}(5)\right)=176
\end{aligned}
$$

and a maximum number of $C^{3}$ self-complementary maximum circular codes MSC ${ }^{3}$ for

$$
\begin{aligned}
\max & \left\{\operatorname{msc}^{3}\left(\mathscr{V}_{\mathrm{II}}^{i}(l)\right), i=1,2,3, l=1, \ldots, 20\right\} \\
& =\operatorname{msc}^{3}\left(\mathscr{V}_{\mathrm{II}}^{2}(4)\right)=6 .
\end{aligned}
$$

\subsubsection{Transversion II Map $\mathscr{V}_{I I}^{i, j}$}

Result 8 (Table 9). For $l=1, \ldots, 20$

$$
\begin{aligned}
& c\left(\mathscr{V}_{\mathrm{II}}^{1,2}(l)\right)=c\left(\mathscr{V}_{\mathrm{II}}^{2,3}(l)\right), \\
& \operatorname{mc}\left(\mathscr{V}_{\mathrm{II}}^{1,2}(l)\right)=\operatorname{mc}\left(\mathscr{V}_{\mathrm{II}}^{2,3}(l)\right), \\
& \operatorname{sc}\left(\mathscr{V}_{\mathrm{II}}^{1,2}(l)\right)=\operatorname{sc}\left(\mathscr{V}_{\mathrm{II}}^{2,3}(l)\right), \\
& c^{3}\left(\mathscr{V}_{\mathrm{II}}^{1,2}(l)\right)=c^{3}\left(\mathscr{V}_{\mathrm{II}}^{2,3}(l)\right), \\
& \operatorname{msc}^{3}\left(\mathscr{V}_{\mathrm{II}}^{1,2}(l)\right)=\operatorname{msc}^{3}\left(\mathscr{V}_{\mathrm{II}}^{2,3}(l)\right) .
\end{aligned}
$$

The lists of trinucleotide circular codes $C$ associated with $c\left(\mathscr{V}_{\mathrm{II}}^{1,2}(l)\right)$ and $c\left(\mathscr{V}_{\mathrm{II}}^{2,3}(l)\right)$ are different for $l=1, \ldots, 5,13,14$ (not shown). The distribution of trinucleotide codes which are not circular under transversions II $\mathscr{V}_{\text {II }}^{i, j}$ in the trinucleotides of the common trinucleotide circular code $X_{0}$ is very unusual. Indeed, for $l=6, \ldots, 12,15, \ldots, 20$

$$
c\left(\mathscr{V}_{\mathrm{II}}^{1,2}(l)\right)=c\left(\mathscr{V}_{\mathrm{II}}^{2,3}(l)\right)=0
$$

and for $l=15, \ldots, 20$

$$
c\left(\mathscr{V}_{\mathrm{II}}^{1,3}(l)\right)=0 .
$$


TABLE 9: Transversion II map $\mathscr{V}_{\text {II }}^{i, j}(l)$ in $l$ trinucleotide of the common trinucleotide circular code $X_{0}$. Number $c\left(\mathscr{V}_{\mathrm{II}}^{i, j}(l)\right)$ of circular codes $C$, number $\operatorname{mc}\left(\mathscr{V}_{\mathrm{II}}^{i, j}(l)\right)$ of maximum circular codes MC, number sc $\left(\mathscr{V}_{\mathrm{II}}^{i, j}(l)\right)$ of self-complementary circular codes $\mathrm{SC}$, number $c^{3}\left(\mathscr{V}_{\mathrm{II}}^{i, j}(l)\right)$ of circular codes $C^{3}$, and number $\operatorname{msc}^{3}\left(\mathscr{V}_{\mathrm{II}}^{i, j}(l)\right)$ of $C^{3}$ self-complementary maximum circular codes MSC ${ }^{3}$.

\begin{tabular}{|c|c|c|c|c|c|c|c|c|c|c|}
\hline \multirow[t]{2}{*}{$l$} & \multicolumn{5}{|c|}{$\mathscr{V}_{\mathrm{II}}^{1,2}(l)$ or $\mathscr{V}_{\mathrm{II}}^{2,3}(l)$} & \multicolumn{5}{|c|}{$\mathscr{V}_{\mathrm{II}}^{1,3}(l)$} \\
\hline & $c$ & $\mathrm{mc}$ & sc & $c^{3}$ & $\mathrm{msc}^{3}$ & $c$ & $\mathrm{mc}$ & sc & $c^{3}$ & $\mathrm{msc}^{3}$ \\
\hline 1 & 5 & 1 & 0 & 5 & 0 & 8 & 0 & 0 & 8 & 0 \\
\hline 2 & 11 & 2 & 3 & 10 & 2 & 40 & 4 & 8 & 30 & 4 \\
\hline 3 & 13 & 2 & 0 & 11 & 0 & 132 & 0 & 0 & 72 & 0 \\
\hline 4 & 8 & 1 & 1 & 7 & 1 & 312 & 6 & 22 & 117 & 6 \\
\hline 5 & 2 & 1 & 0 & 2 & 0 & 532 & 0 & 0 & 130 & 0 \\
\hline 6 & 0 & 0 & 0 & 0 & 0 & 662 & 4 & 24 & 92 & 4 \\
\hline 7 & 0 & 0 & 0 & 0 & 0 & 592 & 0 & 0 & 34 & 0 \\
\hline 8 & 0 & 0 & 0 & 0 & 0 & 389 & 1 & 9 & 4 & 1 \\
\hline 9 & 0 & 0 & 0 & 0 & 0 & 188 & 0 & 0 & 0 & 0 \\
\hline 10 & 0 & 0 & 0 & 0 & 0 & 87 & 0 & 1 & 2 & 0 \\
\hline 11 & 0 & 0 & 0 & 0 & 0 & 54 & 0 & 0 & 6 & 0 \\
\hline 12 & 0 & 0 & 0 & 0 & 0 & 34 & 0 & 2 & 7 & 0 \\
\hline 13 & 1 & 0 & 0 & 0 & 0 & 14 & 0 & 0 & 4 & 0 \\
\hline 14 & 1 & 0 & 0 & 0 & 0 & 3 & 0 & 1 & 3 & 0 \\
\hline$\{15, \ldots, 20\}$ & 0 & 0 & 0 & 0 & 0 & 0 & 0 & 0 & 0 & 0 \\
\hline
\end{tabular}

The transversion II $\mathscr{V}_{\text {II }}^{i, j}$ generates a maximum number of trinucleotide circular codes $C$ for

$$
\begin{aligned}
\max & \left\{c\left(\mathscr{V}_{\mathrm{II}}^{i, j}(l)\right), i, j=1,2,3, i<j, l=1, \ldots, 20\right\} \\
& =c\left(\mathscr{V}_{\mathrm{II}}^{1,3}(6)\right)=662
\end{aligned}
$$

and a maximum number of $C^{3}$ self-complementary maximum circular codes $\mathrm{MSC}^{3}$ for

$$
\begin{aligned}
\max & \left\{\operatorname{msc}^{3}\left(\mathscr{V}_{\mathrm{II}}^{i, j}(l)\right), i, j=1,2,3, i<j, l=1, \ldots, 20\right\} \\
& =\operatorname{msc}^{3}\left(\mathscr{V}_{\mathrm{II}}^{1,3}(4)\right)=6 .
\end{aligned}
$$

The numbers $c\left(\mathscr{V}_{\text {II }}^{1,2}(l)\right)=c\left(\mathscr{V}_{\text {II }}^{2,3}(l)\right)$ of circular codes $C$ have a particular growth function

$$
\begin{array}{cc}
c\left(\mathscr{V}_{\mathrm{II}}^{1,2}(l)\right)=c\left(\mathscr{V}_{\mathrm{II}}^{2,3}(l)\right)=1 & \text { for } l=13,14, \\
c\left(\mathscr{V}_{\mathrm{II}}^{1,2}(l)\right)=c\left(\mathscr{V}_{\mathrm{II}}^{2,3}(l)\right)=0 & \text { for } l=6, \ldots, 12 .
\end{array}
$$

\subsubsection{Transversion II Map $\mathscr{V}_{I I}^{1,2,3}$}

Proposition 37. For $l=1, \ldots, 19$

$$
c\left(\mathscr{V}_{\mathrm{II}}^{1,2,3}(l)\right)=0
$$

and obviously, by letter invariance, $c\left(\mathscr{V}_{\mathrm{II}}^{1,2,3}(20)\right)=1$ as in Tables 4 and 7 .

Proof. The common trinucleotide circular code $X_{0}$ can be partitioned according to the maps $\mathscr{V}_{\mathrm{II}}^{1,2,3}, \mathscr{P}$, and $\mathscr{P}^{2}$ as shown in Table 10.
Let a partition $\mathbf{P}_{i}=\left\{x, x^{\prime}\right\}, i \in\{1, \ldots, 10\}$, composed of two trinucleotides $x, x^{\prime} \in X_{0}$. For $l=1$, any transversion II of a trinucleotide $x \in \mathbf{P}_{i}$ generates a trinucleotide $y$ which is a permuted trinucleotide of the other trinucleotide $x^{\prime} \in \mathbf{P}_{i}$. So, any transversion II of a trinucleotide $x \in X_{0}$ leads to a trinucleotide code which is not circular. For $2 \leq l \leq 19$, the proof needs a computer analysis of the necklace for the nontrivial cases when two transversions II occur with two trinucleotides in the same partitions.

Remark 38. Very surprisingly, for the three maps of transition, transversions I and II, $\mathscr{T}^{i}(l), \mathscr{V}_{\mathrm{I}}^{i}(l)$, and $\mathscr{V}_{\mathrm{II}}^{i}(l), i \in$ $\{1,2,3\}, \mathscr{T}^{i, j}(l), \mathscr{V}_{\mathrm{I}}^{i, j}(l)$, and $\mathscr{V}_{\mathrm{II}}^{i, j}(l), i, j \in\{1,2,3\}$ with $i<j$ and $\mathscr{T}^{1,2,3}(l)$ (not for $\mathscr{V}_{\mathrm{I}}^{1,2,3}(l)$ and $\mathscr{V}_{\mathrm{II}}^{1,2,3}(l)$ ), the numbers $\mathrm{msc}^{3}$ of self-complementary maximum circular codes $\mathrm{MSC}^{3}$ for the first even values of $l$ follow a series of binomial coefficients. For $\mathscr{T}^{i}(l), \mathscr{V}_{\mathrm{I}}^{i}(l)$, and $\mathscr{V}_{\mathrm{II}}^{i}(l), i \in\{1,2,3\}, \mathscr{T}^{1,2}(l)$, $\mathscr{T}^{2,3}(l)$, and $\mathscr{V}_{\text {II }}^{i, j}(l), i, j \in\{1,2,3\}$ with $i<j$, the numbers mc of maximum circular codes MC for the first even values of $l$ follow a series of binomial coefficients. For $\mathscr{T}^{1,2,3}(l)$, the numbers $c^{3}$ of circular codes $C^{3}$ for the values $l$ and $(20-l)$ with $l=1, \ldots, 8$ follow a series of binomial coefficients. These binomial properties with some numbers of circular codes for the three maps of transition, transversions I and II have no combinatorial explanation so far.

\section{Conclusion}

A comprehensive computer analysis of transition and transversions I and II in the $C^{3}$ self-complementary maximum circular code $X_{0}$ shows some new results; in particular (i) transversion $I_{V_{I}}^{2}(l)$ on the 2 nd position of any subset of trinucleotides of $X_{0}$ generates trinucleotide circular codes 
TABLE 10

\begin{tabular}{|c|c|c|c|c|c|}
\hline Partition of $X_{0}$ & $X_{0}$ & $\mathscr{V}_{\mathrm{II}}^{1,2,3}\left(X_{0}\right)=X_{2}$ & $\mathscr{P}\left(X_{2}\right)=X_{0}^{\prime}$ & $\mathscr{V}_{\mathrm{II}}^{1,2,3}\left(X_{0}^{\prime}\right)=X_{1}$ & $\mathscr{P}^{2}\left(X_{1}\right)=X_{0}$ \\
\hline $\mathbf{P}_{1}=\{A A C, A C C\}$ & $A C C$ & $C A A$ & $A A C$ & $C C A$ & $A C C$ \\
\hline $\mathbf{P}_{2}=\{A A T, G C C\}$ & GCC & TAA & $A A T$ & $C C G$ & GCC \\
\hline $\mathbf{P}_{3}=\{A T C, G A C\}$ & ATC & $C G A$ & $G A C$ & TCA & ATC \\
\hline $\mathbf{P}_{4}=\{A T T, G G C\}$ & $A T T$ & $C G G$ & GGC & TTA & $A T T$ \\
\hline $\mathbf{P}_{5}=\{C A G, T A C\}$ & TAC & $G C A$ & $C A G$ & $A C T$ & TAC \\
\hline $\mathbf{P}_{6}=\{C T C, G A A\}$ & CTC & $A G A$ & GAA & TCC & CTC \\
\hline $\mathbf{P}_{7}=\{C T G, G T A\}$ & $C T G$ & $A G T$ & GTA & TGC & CTG \\
\hline $\mathbf{P}_{8}=\{G A G, T T C\}$ & TTC & $G G A$ & $G A G$ & TCT & TTC \\
\hline $\mathbf{P}_{9}=\{G A T, G T C\}$ & GTC & $T G A$ & GAT & TCG & GTC \\
\hline $\mathbf{P}_{10}=\{G G T, G T T\}$ & $G T T$ & $T G G$ & GGT & TTG & GTT \\
\hline
\end{tabular}

which are always $C^{3}$ and (ii) transversion II $\mathscr{V}_{\text {II }}^{1,2,3}$ on the three positions of any subset of trinucleotides of $X_{0}$ yields no trinucleotide circular codes. In addition to the classical self-complementary (Definition 20) partition of $X_{0}$ known since 1996, a new partition of $X_{0}$ based on the transversion II map $\mathscr{V}_{\text {II }}^{1,2,3}$ (Definition 33) and the circular permutation maps $\mathscr{P}$ and $\mathscr{P}^{2}$ (Definition 18) is also identified here. These results here extend our theory of circular code in genes to its evolution under transition and transversion.

\section{References}

[1] C. J. Michel, G. Pirillo, and M. A. Pirillo, "A relation between trinucleotide comma-free codes and trinucleotide circular codes," Theoretical Computer Science, vol. 401, no. 1-3, pp. 1726, 2008.

[2] C. J. Michel and G. Pirillo, "Identification of all trinucleotide circular codes," Computational Biology and Chemistry, vol. 34, no. 2, pp. 122-125, 2010.

[3] L. Bussoli, C. J. Michel, and G. Pirillo, "On some forbidden configurations for self-complementary trinucleotide circular codes," Journal for Algebra and Number Theory Academia, vol. 2, pp. 223-232, 2011.

[4] L. Bussoli, C. J. Michel, and G. Pirillo, "On conjugation partitions of sets of trinucleotides," Applied Mathematics, vol. 3, pp. 107-112, 2012.

[5] C. J. Michel, G. Pirillo, and M. A. Pirillo, "A classiffication of 20-trinucleotide circular codes," Information and Computation, vol. 212, pp. 55-63, 2012.

[6] C. J. Michel, G. Pirillo, and M. A. Pirillo, "Varieties of commafree codes," Computers and Mathematics with Applications, vol. 55, no. 5, pp. 989-996, 2008.

[7] C. J. Michel and G. Pirillo, "Strong trinucleotide circular codes," International Journal of Combinatorics, vol. 2011, Article ID 659567, 14 pages, 2011.

[8] D. G. Arquès and C. J. Michel, "A complementary circular code in the protein coding genes," Journal of Theoretical Biology, vol. 182, no. 1, pp. 45-58, 1996.

[9] D. L. Gonzalez, S. Giannerini, and R. Rosa, "Circular codes revisited: a statistical approach," Journal of Theoretical Biology, vol. 275, pp. 21-28, 2011.

[10] C. J. Michel, "Circular code motifs in transfer and 16S ribosomal RNAs: a possible translation code in genes," Computational Biology and Chemistry, vol. 37, pp. 24-37, 2012.
[11] F. H. C. Crick, J. S. Griffith, and L. E. Orgel, "Codes without commas," Proceedings of the National Academy of Sciences, vol. 43, pp. 416-421, 1957.

[12] S. W. Golomb, B. Gordon, and L. R. Welch, "Comma-free codes," Canadian Journal of Mathematics, vol. 10, pp. 202-209, 1958.

[13] S. W. Golomb, L. R. Welch, and M. Delbrück, "Construction and properties of comma-free codes," in Biologiske Meddelelser, vol. 23, Det Kongelige Danske Videnskabernes Selskab, 1958.

[14] B. Hayes, "The Invention of the Genetic Code," American Scientist, vol. 86, no. 1, pp. 8-14, 1998.

[15] A. J. Koch and J. Lehmann, "About a symmetry of the genetic code," Journal of Theoretical Biology, vol. 189, no. 2, pp. 171-174, 1997.

[16] R. Jolivet and F. Rothen, "Peculiar symmetry of DNA sequences and evidence suggesting its evolutionary origin in a primeval genetic code," in Proceedings of the 1st European Workshop Exo-/Astrobiology, P. Ehrenfreund, O. Angerer, and B. Battrick, Eds., ESA SP-496, pp. 173-176, May 2001.

[17] C. Nikolaou and Y. Almirantis, "Mutually symmetric and complementary triplets: differences in their use distinguish systematically between coding and non-coding genomic sequences," Journal of Theoretical Biology, vol. 223, no. 4, pp. 477-487, 2003.

[18] G. Pirillo, "A characterization for a set of trinucleotides to be a circular code," in Determinism, Holism, and Complexity, C. Pellegrini, P. Cerrai, P. Freguglia, V. Benci, and G. Israel, Eds., Kluwer, 2003.

[19] G. Pirillo and M. A. Pirillo, "Growth function of selfcomplementary circular codes," Rivista di Biologia, vol. 98, no. 1, pp. 97-110, 2005.

[20] J. L. Lassez, R. A. Rossi, and A. E. Bernal, "Crick's hypothesis revisited: the existence of a universal coding frame," in Proceedings of the IEEE 21st International Conference on Advanced Information Networking and Applications Workshops (AINAW '07), May 2007.

[21] G. Pirillo, "A hierarchy for circular codes," RAIRO: Theoretical Informatics and Applications, vol. 42, no. 4, pp. 717-728, 2008.

[22] M. Kimura, "Estimation of evolutionary distances between homologous nucleotide sequences," Proceedings of the National Academy of Sciences of the United States of America, vol. 78, no. 1, pp. 454-458, 1981.

[23] J. Berstel and D. Perrin, Theory of Codes, Academic Press, London, UK, 1985.

[24] J. L. Lassez, "Circular codes and synchronization," International Journal of Computer and Information Sciences, vol. 5, no. 2, pp. 201-208, 1976. 

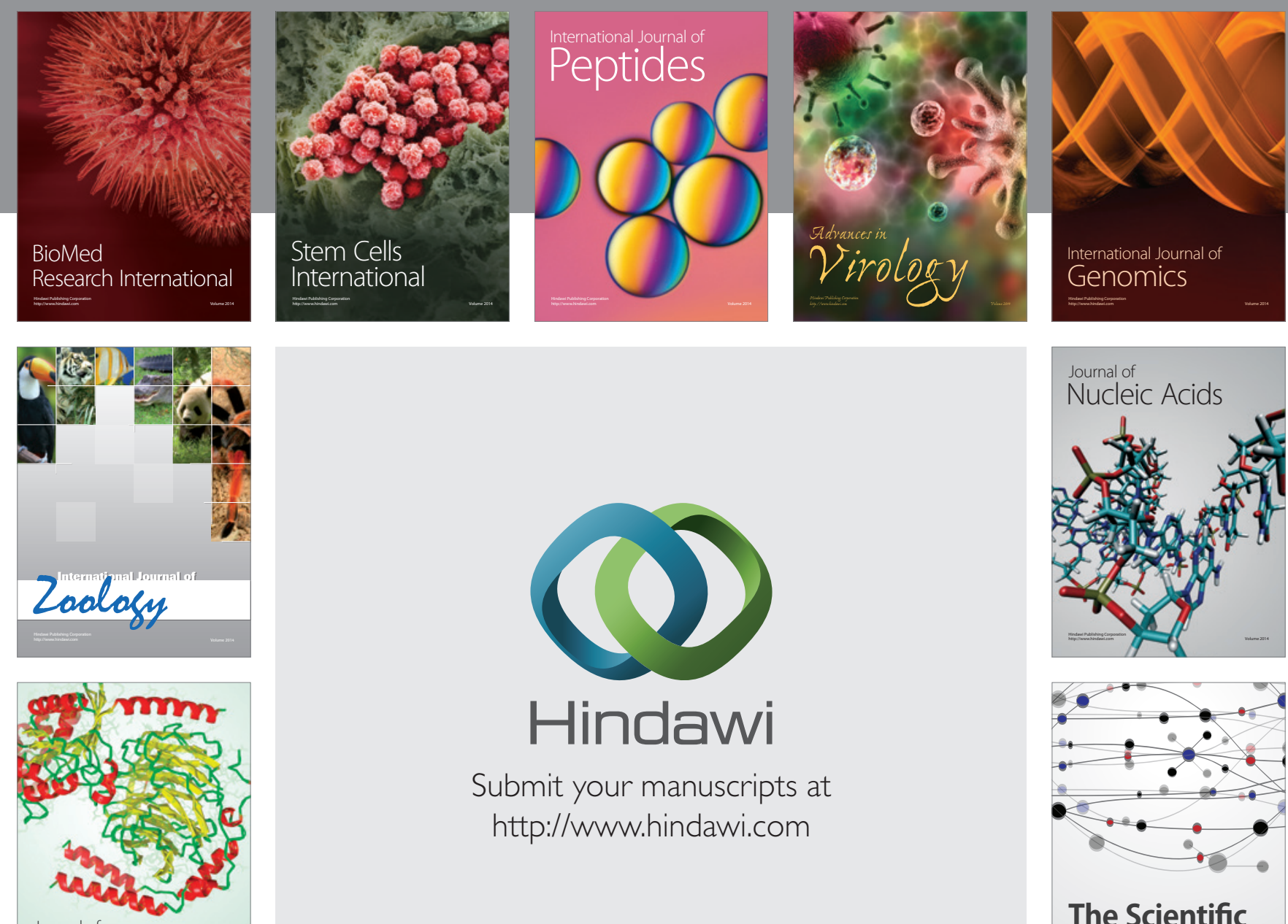

Submit your manuscripts at

http://www.hindawi.com

Journal of
Signal Transduction
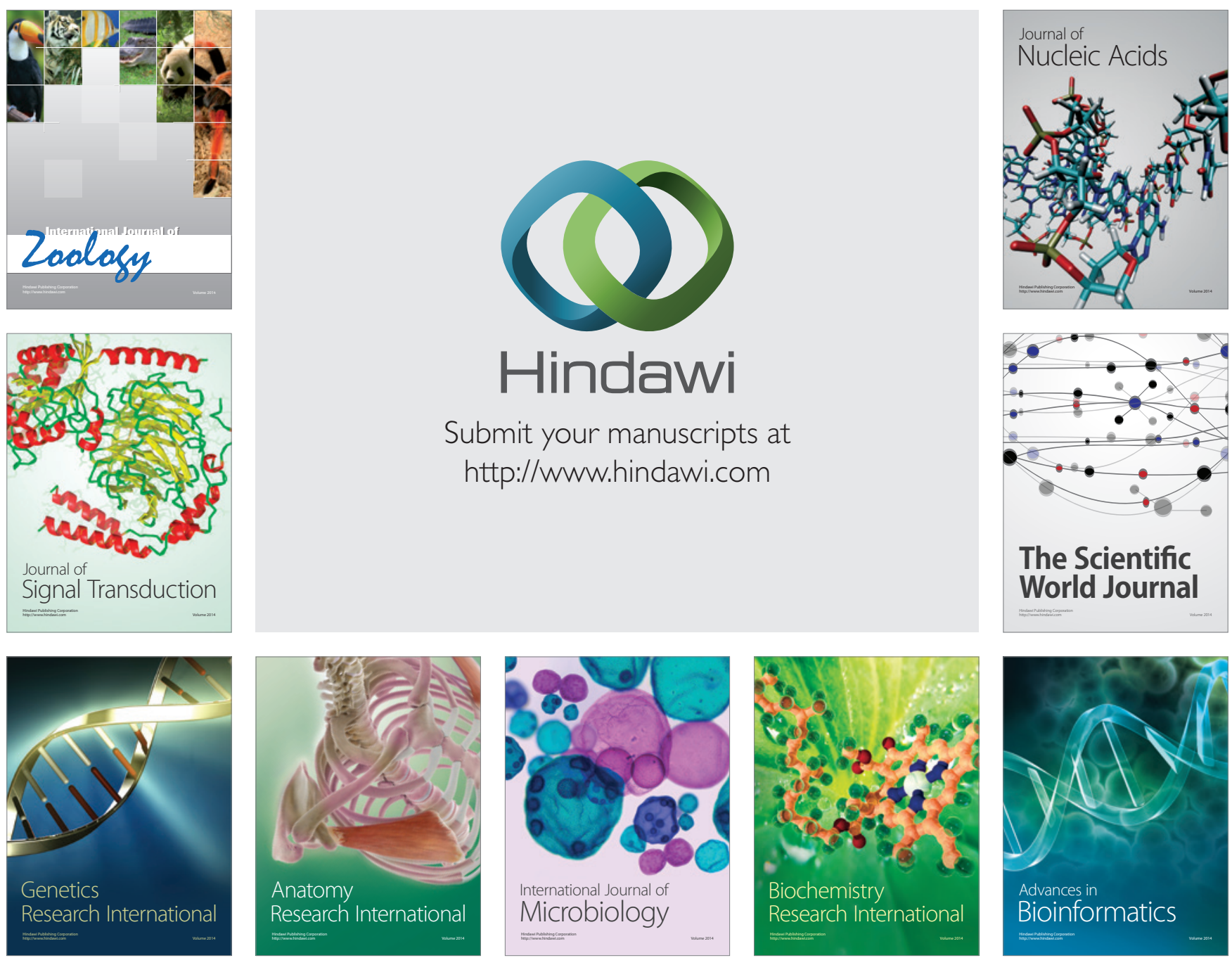

The Scientific World Journal
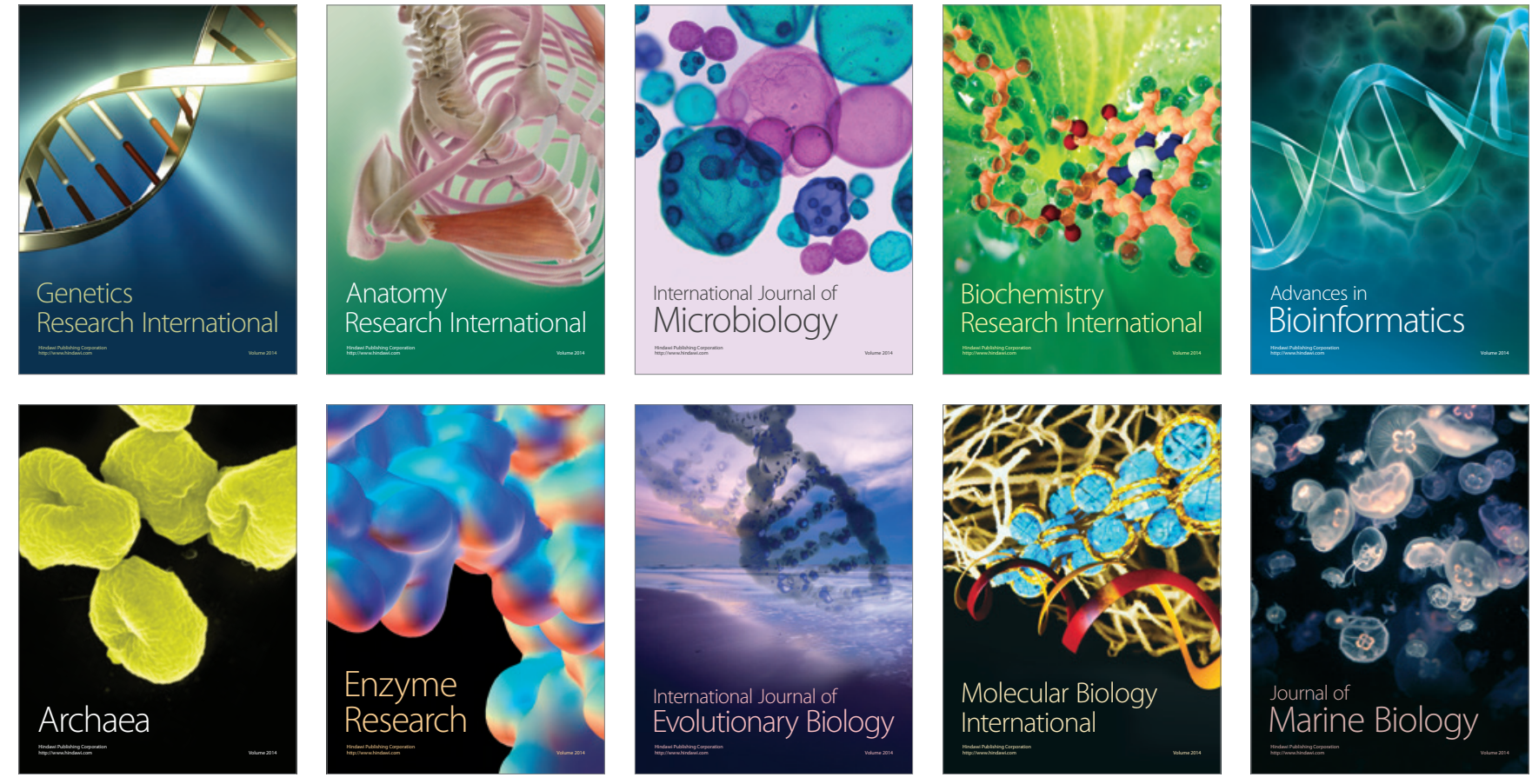